NBI-HE-00-28

hep-th/0006023

June, 2000

\title{
Supergravity and Space-Time Non-Commutative Open String Theory
}

\author{
Troels Harmark円 \\ The Niels Bohr Institute \\ Blegdamsvej 17, DK-2100 Copenhagen Ø, Denmark
}

\begin{abstract}
We study the non-critical space-time non-commutative open string (NCOS) theory using a dual supergravity description in terms of a certain near-horizon limit of the F1-D $p$ bound state. We find the thermodynamics of NCOS theory from supergravity. The thermodynamics is equivalent to Yang-Mills theory on a commutative space-time. We argue that this fact does not have to be in contradiction with the expected Hagedorn behaviour of NCOS theory. To support this we consider string corrections to the thermodynamics. We also discuss the relation to Little String Theory in 6 dimensions.
\end{abstract}

\footnotetext{
${ }^{1}$ e-mail: harmark@nbi.dk
} 


\section{Introduction}

String theory in the presence of $\mathrm{D} p$-branes with a magnetic NSNS $B$-field flux gives a $p+1$ dimensional Yang-Mills theory on a non-commutative space (NCYM) in a certain decoupling limit [1, 2]. Specifically, the coordinates of NCYM has the commutator

$$
\left[x^{i}, x^{j}\right]=i \theta^{i j}
$$

Recently, it has been shown that one also can obtain a theory with space and time non-commuting from string theory [3, 4$]^{2}$. Thus, it has the commutator

$$
\left[x^{0}, x^{i}\right]=i \theta^{0 i}
$$

The new $p+1$ dimensional theory with this commutator is obtained from the decoupling limit of $\mathrm{D} p$-branes in the presence of a near-critical electrical NSNS $B$-field [3, [4]. The theory thus obtained is not a field theory but a $p+1$ dimensional space-time non-commutative open string (NCOS) theory [3, 田陷 NCOS theory is a new non-critical supersymmetric string theory which is believed to be non-gravitational and not to have a closed string sector [3, 田].

In this paper we study NCOS theory from supergravity. Our starting point is the conjecture that $p+1$ dimensional NCOS theory is dual to string theory on the background given by a certain near-horizon limit of the F1-D $p$ bound state [4] In [1] the near-horizon limit of the F1-D3 bound state was constructed, and we generalize this to non-extremal F1-D $p$ bound states. This adds to the list of correspondences between string theory in the presence of $\mathrm{D} p$-branes with a non-zero NSNS $B$-field and dual non-gravitational theories, which has been studied for magnetic NSNS $B$-field backgrounds in [12, 13, 14, 15, 16, 17, 18.

We use the near-horizon background to find the thermodynamics of NCOS theory and we show that it is equivalent to the thermodynamics of ordinary Yang-Mills (OYM) theory, that is, Yang-Mills theory on a commutative spacetime. The main goal of this paper is thus to understand how this thermodynamics can be consistent with the expectation that NCOS theory is a string theory and therefore should have Hagedorn behaviour.

\footnotetext{
${ }^{2}$ See [5, [6] for other recent work on space-time non-commutativity in string theory.

${ }^{3}$ That the theory with space and time non-commuting is not a field theory could seem surprising since (2) could be viewed as the Lorentz-invariant completion of (1). But this is explained by the fact that field theories with space and time being non-commutative are not unitary and thus cannot be a consistent truncation of string theory [7].

${ }^{4}$ See [8] for other recent work on NCOS theory.

${ }^{5}$ This is in the spirit of the near-horizon-D $p$-brane/QFT correspondence [9, 10, 11]
} 
We find that the thermodynamics obtained from supergravity can be consistent with Hagedorn behaviour of NCOS theory. We find a region where the NCOS string coupling is very weak and where the temperature is of the same order as the NCOS Hagedorn temperature, and by analyzing the phases of the supergravity description we find that this region cannot be described by supergravity. In other words, we find that one cannot both be near the Hagedorn temperature and have arbitrarily weak coupling in the supergravity description of NCOS theory.

By analyzing the high energy behaviour of the NCOS supergravity description we find that it can be described by delocalized F-strings. This we use to approach the region mentioned above by doing tree-level string corrections to the thermodynamics, and we find that in this region NCOS theory has thermodynamics different from that of OYM. This supports our conclusions that the supergravity thermodynamics is consistent with NCOS Hagedorn behaviour.

We also consider 6 dimensional NCOS theory and the relation to space-time non-commutative Little String Theory. This is interesting since this gives us two non-critical string theories which should be related to each other.

This paper is organized as follows. We give the non-extremal F1-D $p$ bound state in Section 2. We then take the near-horizon limit in Section 3 and thereby obtain the background solution that corresponds to finite temperature NCOS theory. The phase structure of the supergravity description is studied in Section 1 and the NCOS thermodynamics is found and discussed in Section 5. We then show explicitly that the near-horizon background dual to NCOS theory reduces to delocalized F-strings in Section 6. This is used in Section 7 to search for a region with new thermodynamics of the 4 dimensional NCOS theory. We end by discussing the 6 dimensional NCOS theory and its relation to Little String Theory in Section 8 .

\section{The non-extremal F1-D $p$ bound state}

In this section we present the non-extremal F1-D $p$ bound state along with its thermodynamics ${ }^{0}$. In the extremal limit it reduces to the extremal F1-D $p$ bound state given in 20, 21, 22, 23].

The non-extremal F1-D3 bound state can be obtained by S-duality from the

\footnotetext{
${ }^{6}$ Non-extremal generalizations of non-threshold brane solutions were first considered in [19.
} 
non-extremal D1-D3 bound stateף. By use of T-duality on the non-extremal F1-D3 bound state we obtain the non-extremal F1-D $p$ bound state with the string frame metric

$$
\begin{aligned}
d s^{2}= & \hat{D}^{-1 / 2} \hat{H}^{-1 / 2}\left[-f d t^{2}+\left(d x^{1}\right)^{2}\right]+\hat{D}^{1 / 2} \hat{H}^{-1 / 2}\left[\left(d x^{2}\right)^{2}+\cdots+\left(d x^{p}\right)^{2}\right] \\
& +\hat{D}^{-1 / 2} \hat{H}^{1 / 2}\left[f^{-1} d r^{2}+r^{2} d \Omega_{8-p}^{2}\right]
\end{aligned}
$$

the dilaton

$$
e^{2 \phi}=\hat{D}^{\frac{p-5}{2}} \hat{H}^{\frac{3-p}{2}}
$$

and the potentials

$$
\begin{aligned}
B_{t 1} & =\sin \hat{\theta}\left(\hat{H}^{-1}-1\right) \operatorname{coth} \hat{\alpha} \\
A_{2 \cdots p} & =(-1)^{p} \tan \hat{\theta}\left(\hat{H}^{-1} \hat{D}-1\right) \\
A_{t 1 \cdots p} & =(-1)^{p} \cos \hat{\theta} \hat{D}\left(\hat{H}^{-1}-1\right) \operatorname{coth} \hat{\alpha}
\end{aligned}
$$

where $B_{\mu \nu}$ is the NSNS two-form, and $A_{\mu_{1} \cdots \mu_{p-1}}$ and $A_{\mu_{1} \cdots \mu_{p+1}}$ are the RR $(p-1)$-form and $(p+1)$-form potentials?. We have also

$$
\begin{gathered}
\hat{H}=1+\frac{r_{0}^{7-p} \sinh ^{2} \hat{\alpha}}{r^{7-p}}, \quad f=1-\frac{r_{0}^{7-p}}{r^{7-p}} \\
\hat{D}^{-1}=\cos ^{2} \hat{\theta}+\sin ^{2} \hat{\theta} \hat{H}^{-1}
\end{gathered}
$$

The thermodynamics is given by

$$
\begin{gathered}
M=\frac{V_{p} V\left(S^{8-p}\right)}{16 \pi G} r_{0}^{7-p}\left[8-p+(7-p) \sinh ^{2} \hat{\alpha}\right] \\
T=\frac{7-p}{4 \pi r_{0} \cosh \hat{\alpha}}, \quad S=\frac{V_{p} V\left(S^{8-p}\right)}{4 G} r_{0}^{8-p} \cosh \hat{\alpha} \\
\mu_{\mathrm{F} 1}=-\sin \hat{\theta} \tanh \hat{\alpha}, \quad Q_{\mathrm{F} 1}=-\sin \hat{\theta} \frac{V_{p} V\left(S^{8-p}\right)}{16 \pi G}(7-p) r_{0}^{7-p} \cosh \hat{\alpha} \sinh \hat{\alpha}
\end{gathered}
$$

\footnotetext{
${ }^{7}$ The non-extremal D1-D3 bound state can be found in [16] in a similar notation as this paper. One can then do the S-duality transformation $g_{\mu \nu}^{E} \rightarrow g_{\mu \nu}^{E}, e^{\phi} \rightarrow e^{-\phi}, B_{\mu \nu} \rightarrow A_{\mu \nu}$, $A_{\mu \nu} \rightarrow-B_{\mu \nu}, A_{\mu \nu \rho \sigma} \rightarrow A_{\mu \nu \rho \sigma}$ on the D1-D3 bound state where $g_{\mu \nu}^{E}$ refers to the Einsteinframe metric.

${ }^{8}$ The RR five-form field strength obtained from the RR four-form potential should be made self-dual.
} 


$$
\mu_{\mathrm{D} p}=\cos \hat{\theta} \tanh \hat{\alpha} \quad, \quad Q_{\mathrm{D} p}=\cos \hat{\theta} \frac{V_{p} V\left(S^{8-p}\right)}{16 \pi G}(7-p) r_{0}^{7-p} \cosh \hat{\alpha} \sinh \hat{\alpha}
$$

We note that just as for the D-brane bound state solutions the thermodynamics only depends on the angle $\hat{\theta}$ in the the charges and chemical potentialsf? We also note that for the F1-D3 bound state we have the same thermodynamics as the D1-D3 bound state which reflects the fact that these two bound states are S-dual to each other.

Using charge quantization of the $\mathrm{D} p$-brane we get

$$
r_{0}^{7-p} \cosh \hat{\alpha} \sinh \hat{\alpha}=\frac{(2 \pi)^{7-p} g_{s} N l_{s}^{7-p}}{(7-p) V\left(S^{8-p}\right) \cos \hat{\theta}}
$$

where $N$ is the number of coincident $\mathrm{D} p$-branes. The angle $\hat{\theta}$ is related to the number $M$ of F-strings in the bound state as

$$
\tan \hat{\theta}=-\frac{Q_{\mathrm{F} 1}}{Q_{\mathrm{D} p}}=\frac{V_{1} T_{\mathrm{F} 1} M}{V_{p} T_{\mathrm{D} p} N}=g_{s} \frac{\left(2 \pi l_{s}\right)^{p-1}}{V_{p-1}} \frac{M}{N}
$$

Here $V_{p}=\int d x^{1} \cdots d x^{p}, V_{1}=\int d x^{1}$ and $V_{p-1}=\int d x^{2} \cdots d x^{p}$. We now go to another choice of variables for the solution (3)-(7) which for our purposes are more natural. We introduce the variables $\alpha$ and $\theta$ as

$$
\sinh ^{2} \alpha=\cos ^{2} \hat{\theta} \sinh ^{2} \hat{\alpha}, \quad \cosh ^{2} \theta=\frac{1}{\cos ^{2} \hat{\theta}}
$$

In terms of the variables (16) we can write the metric, dilaton and NSNS $B$-field as

$$
\begin{gathered}
d s^{2}=H^{-1 / 2}\left[D\left(-f d t^{2}+\left(d x^{1}\right)^{2}\right)+\left(d x^{2}\right)^{2}+\cdots+\left(d x^{p}\right)^{2}\right] \\
+H^{1 / 2}\left[f^{-1} d r^{2}+r^{2} d \Omega_{8-p}^{2}\right] \\
e^{2 \phi}=H^{\frac{3-p}{2}} D \\
B_{t 1}=\tanh \theta \sqrt{1+\cosh ^{-2} \theta \sinh ^{-2} \alpha}\left(D H^{-1}-1\right)
\end{gathered}
$$

with

$$
H=1+\frac{r_{0}^{7-p} \sinh ^{2} \alpha}{r^{7-p}}, \quad D^{-1}=\cosh ^{2} \theta-\sinh ^{2} \theta H^{-1}
$$

\footnotetext{
${ }^{9}$ See [16 for an explicit account of this.
} 
We see that the solution (17)-(20) are similar in form to that of the $\mathrm{D}(p-2)$ D $p$ bound state with a NSNS $B$ field flux turned on, only here the $B$ field is turned on in an electrical component rather than a magnetic component. For the extremal F1-D $p$ bound state one can in fact obtain the solution by Wickrotating the Lorentzian D $p$-brane solution into an Euclidean solution and then use T-duality and rotations in the same manner as for magnetic $B$ fields, in order to turn an electrical $B$-field component on[12]. After Wick rotating back to a Lorentzian solution one then has the F1-D $p$ bound state. This, however, does not work in the more general case of a non-extremal F1-D $p$ bound state. Here one cannot obtain the F1-D $p$ bound state from the $\mathrm{D} p$-brane by T-duality and rotations. This is due to subtleties occuring when doing T-duality in an Euclidean time-direction 24].

In the new variables (16) the thermodynamics (10)-(13) is given by

$$
\begin{aligned}
M & =\frac{V_{p} V\left(S^{8-p}\right)}{16 \pi G} r_{0}^{7-p}\left[8-p+(7-p) \cosh ^{2} \theta \sinh ^{2} \alpha\right] \\
T & =\frac{7-p}{4 \pi r_{0} \sqrt{1+\cosh ^{2} \theta \sinh ^{2} \alpha}} \\
S & =\frac{V_{p} V\left(S^{8-p}\right)}{4 G} r_{0}^{8-p} \sqrt{1+\cosh ^{2} \theta \sinh ^{2} \alpha} \\
\mu_{\mathrm{F} 1} & =-\frac{\sinh \theta \sinh \alpha}{\sqrt{1+\cosh ^{2} \theta \sinh ^{2} \alpha}} \\
Q_{\mathrm{F} 1} & =-\frac{V_{p} V\left(S^{8-p}\right)}{16 \pi G}(7-p) r_{0}^{7-p} \sinh \theta \sinh \alpha \sqrt{1+\cosh ^{2} \theta \sinh ^{2} \alpha} \\
\mu_{\mathrm{D} p} & =\frac{\sinh ^{2}}{\sqrt{1+\cosh ^{2} \theta \sinh ^{2} \alpha}} \\
Q_{\mathrm{D} p} & =\frac{V_{p} V\left(S^{8-p}\right)}{16 \pi G}(7-p) r_{0}^{7-p} \sinh \alpha \sqrt{1+\cosh ^{2} \theta \sinh ^{2} \alpha}
\end{aligned}
$$

The charge quantization relation (14) becomes

$$
r_{0}^{7-p} \sinh \alpha \sqrt{\sinh ^{2} \alpha+\cosh ^{-2} \theta}=\frac{(2 \pi)^{7-p} g_{s} N l_{s}^{7-p}}{(7-p) V\left(S^{8-p}\right) \cosh \theta}
$$

\section{The NCOS near-horizon limit}

In this section we apply the limit found in $[4$ to the non-extremal F1-D $p$ bound state. This gives a dual string theory description of the $p+1$ dimensional NCOS theory in terms of string theory on a curved background space-time in the presence of a near-critical electrical NSNS $B$ field. As explained in [3, 4] 
the near-critical electrical NSNS $B$ field gives dynamical open string modes in the decoupled theory since the open string tension almost cancels with the $B$ field giving a finite effective open string tension.

Following [4], we take the limit $l_{s} \rightarrow 0$ keeping fixed

$$
u=\frac{r}{l_{s}}, \quad u_{0}=\frac{r_{0}}{l_{s}}, \quad b=l_{s}^{2} \cosh \theta, \quad \alpha=\text { fixed }, \quad \tilde{g}=\frac{g_{s} l_{s}^{2}}{b}
$$

Moreover, we rescale the world-volume coordinates

$$
\tilde{x}^{i}=\frac{l_{s}}{b} x^{i}, i=0,1, \quad \tilde{x}^{j}=\frac{1}{l_{s}} x^{j}, i=2, \ldots, p
$$

with the notation $t=x^{0}$. Notice that we keep $u=r / l_{s}$ finite since the mass of an open string stretched between to D-branes with distance $r$ measured in units of $l_{s}^{-1}$ is $l_{s} M \sim u$. Thus, contrary to the usual scaling of the radial coordinate $u=r / l_{s}^{2}$ we do not let the string modes measured in units of $l_{s}^{-1}$ grow to infinity, but instead keep them finite and thereby dynamical.

We note that the rescaling of coordinates in (29) and (30) makes all coordinates dimensionless. This means that when measuring distance or time on the world-volume with the coordinates $\tilde{t}, \tilde{x}^{1}, \ldots, \tilde{x}^{p}$ we are measuring in units of $\sqrt{b}$, and similarly we measure energy in units of $1 / \sqrt{b}$.

Defining

$$
R^{7-p} \equiv \frac{(2 \pi)^{7-p}}{(7-p) V\left(S^{8-p}\right)} \tilde{g} N
$$

we have from (28) that

$$
u_{0}^{7-p} \sinh ^{2} \alpha=R^{7-p}
$$

Using the near-horizon limit defined by (29)-(30) on (17) and (18) we get the string-frame metric and dilaton

$$
\begin{gathered}
\frac{d s^{2}}{l_{s}^{2}}=H^{1 / 2} \frac{u^{7-p}}{R^{7-p}}\left[-f d \tilde{t}^{2}+\left(d \tilde{x}^{1}\right)^{2}\right]+H^{-1 / 2}\left[\left(d \tilde{x}^{2}\right)^{2}+\cdots+\left(d \tilde{x}^{p}\right)^{2}\right] \\
+H^{1 / 2}\left[f^{-1} d u^{2}+u^{2} d \Omega_{8-p}^{2}\right] \\
g_{s}^{2} e^{2 \phi}=\tilde{g}^{2}\left(1+\frac{u^{7-p}}{R^{7-p}}\right) H^{\frac{3-p}{2}}
\end{gathered}
$$

where

$$
H=1+\frac{R^{7-p}}{u^{7-p}}
$$


From (19) we get the NSNS $B$-field

$$
B_{t 1}=l_{s}^{2} \frac{u^{7-p}}{R^{7-p}}
$$

We note that the constant appearing in the NSNS $B$-field in (19) has been gauged away before taking the limit. This gives a non-zero electrical component for the world-volume field strength $F_{01}$ on the $\mathrm{D} p$-branes. The limit $l_{s} \rightarrow 0$ keeping fixed (29) and (30) corresponds to approaching the critical value of the electrical field $F_{01}$.

\section{Phases of the supergravity description}

We now analyze the phases of the supergravity near-horizon solution (33)-(36).

The F1-D $p$ bound state in the limit (29) and (30) is believed to describe the $p+1$ dimensional NCOS theory. The open strings of NCOS theory live in a $p+1$ dimensional space-time with the time $\tilde{t}$ and the space-direction $\tilde{x}^{1}$

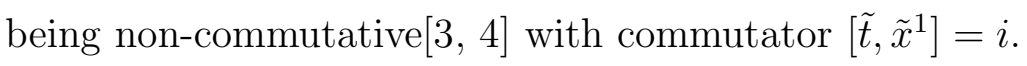

The string length in NCOS theory is $\sqrt{b}$ [3], 4], so since we measure length in units of $\sqrt{b}$ the NCOS string length in our supergravity description is 1 . Thus, if there is any Hagedorn behaviour of the thermodynamics it should occur at $T \sim 1$. The open string coupling constant of NCOS theory is $G_{\mathrm{o}}$ which is related to $\tilde{g}$ as $\tilde{g}=G_{\mathrm{o}}^{2}[4]$. The rescaled radial parameter $u$ is the energy of the brane probe in units of $1 / \sqrt{b}$. Thus $u$ is the effective energy scale in NCOS theory.

It is easily seen that for $u \ll R$ the near-horizon solution reduces to that of the ordinary near-horizon limit of $\mathrm{D} p$-branes (see for example [10]). Thus, for these energies the NCOS theory reduces to an ordinary Yang-Mills (OYM) theory in a $p+1$ dimensional commutative space-time. One can therefore regard OYM as a low energy effective theory for NCOS theory. $u \sim R$ is the energy scale where the non-commutativity start appearing. Since NCOS theory reduces to OYM for $u \ll R$ we define the effective coupling constant

$$
g_{\text {eff }}^{2}=(2 \pi)^{p-2} \tilde{g} N u^{p-3}
$$

This coupling constant corresponds to the usual effective coupling constant for OYM defined in [10] with $g_{\mathrm{YM}}^{2}=(2 \pi)^{p-2} \tilde{g}$ the Yang-Mills coupling constant and $N$ the rank of $U(N)$. Note that $g_{\text {eff }}^{2} \sim R^{7-p} u^{p-3}$. 
For $u \gg R$ the near-horizon solution instead reduces to being the nearhorizon limit of delocalized F-strings. This will be further explained and studied in Section 6. Since $u \sim R$ is the point where we go from the near-horizon $\mathrm{D} p$-brane phase to the near-horizon delocalized F-string phase, we expect that this is the point where the non-commutative effects become significant, in analogy with the near-horizon $\mathrm{D}(p-2)$ - $\mathrm{D} p$ description of NCYM.

The curvature of (33) in units of $l_{s}$ is

$$
\mathcal{C}=\frac{1}{\sqrt{u^{4}+R^{7-p} u^{p-3}}}
$$

In order for the near-horizon solution (33)-(36) to accurately describe NCOS theory we need both $\mathcal{C} \ll 1$ and $g_{s} e^{\phi} \ll 1$. We now analyze these conditions in the two cases $u \ll R$ and $u \gg R$.

If we consider $u \ll R$ where the dual theory is OYM, we see that $\mathcal{C} \ll 1$ is equivalent to having $g_{\mathrm{eff}}^{2} \gg 1$. Thus, we have the usual demand that the effective coupling should be large. The condition $g_{s} e^{\phi} \ll 1$ is equivalent to $g_{\text {eff }}^{2} \ll N^{\frac{4}{7-p}}$, thus we get

$$
1 \ll g_{\text {eff }}^{2} \ll N^{\frac{4}{7-p}}
$$

so that $N \gg 1$ which means we are in the planar limit of OYM. The condition (39) corresponds to the standard supergravity description of OYM given in [9, 10]. Combining the condition $u \ll R$ with (39) we get the additional conditions that $\tilde{g} \gg 1 / N$ for $p>3,1 / N \ll \tilde{g} \ll 1$ for $p=3$ and $\tilde{g} \ll 1$ for $p<3$.

Considering instead $u \gg R$ we see that $g_{s} e^{\phi} \ll 1$ if

$$
u^{7-p} \ll \frac{R^{7-p}}{\tilde{g}^{2}} \sim \frac{N}{\tilde{g}}
$$

Combining this with $u \gg R$ we get that $\tilde{g} \ll 1$ which means that the nearhorizon solution describes weakly coupled NCOS theory. The bound $u^{7-p} \ll$ $N / \tilde{g}$ means that for larger energies the theory flows to the S-dual near-horizon brane description. For $p=3$ we go to NCYM described by the near-horizon D1-D3 solution 25, 4. We discuss the case $p=5$ in Section 8.

Demanding $\mathcal{C} \ll 1$ for $u \gg R$ we see from (38) that we need $u \gg 1$. This we can split in two cases. If $R \gg 1$, which is equivalent to $\tilde{g} \gg 1 / N$, we only need to have $u \gg R$. If $R \ll 1$, which is equivalent to $\tilde{g} \ll 1 / N$, we see that we need to have $u \gg 1$ instead. 
Thus, if we want to consider NCOS theory with $p \geq 3$ when $\tilde{g} \ll 1 / N$ we need to have $u \gg 1$. This means that there is an energy range $R \ll u \ll 1$ in which we cannot describe NCOS theory with arbitrarily weak coupling. This will be important for our discussion of thermodynamics in Section 5 . For completeness we note that for $p<3$ we can also have $\tilde{g} \ll 1 / N$ if $u \ll$ $(\tilde{g} N)^{1 /(3-p)}$ which means that we are in the OYM region.

We can also ask if it possible to find a regime with finite $N$. From the previous paragraph we immediately get that this is possible if and only if $u \gg 1$.

\section{NCOS thermodynamics}

Using the limit (29)-(30) we have from (21)-(27) the thermodynamics

$$
\begin{gathered}
T=\frac{7-p}{4 \pi u_{0}}\left(\frac{u_{0}}{R}\right)^{\frac{7-p}{2}}, \quad S=\frac{\tilde{V}_{p} V\left(S^{8-p}\right)}{32 \pi^{6} \tilde{g}^{2}} u_{0}^{8-p}\left(\frac{R}{u_{0}}\right)^{\frac{7-p}{2}} \\
E=\frac{\tilde{V}_{p} V\left(S^{8-p}\right)}{128 \pi^{7} \tilde{g}^{2}} \frac{9-p}{2} u_{0}^{7-p}, \quad F=-\frac{\tilde{V}_{p} V\left(S^{8-p}\right)}{128 \pi^{7} \tilde{g}^{2}} \frac{5-p}{2} u_{0}^{7-p}
\end{gathered}
$$

with

$$
\tilde{V}_{p}=b^{-1} l_{s}^{2-p} V_{p}
$$

being the world-volume in the rescaled coordinates given by (30). The energy $E$ is the energy above extremality $E=M-\sqrt{Q_{\mathrm{F} 1}^{2}+Q_{\mathrm{D} p}^{2}}$. We note that in order to obtain (41) and (42) it is necessary to rescale $T, E$ and $F$ as

$$
T \rightarrow \frac{b}{l_{s}} T, \quad E \rightarrow \frac{b}{l_{s}} E, \quad F \rightarrow \frac{b}{l_{s}} F
$$

since we have rescaled the time as $\tilde{t}=t l_{s} / b$.

The curvature in units of $l_{s}$ at the horizon is

$$
\varepsilon_{D}=\frac{1}{\sqrt{u_{0}^{4}+R^{7-p} u_{0}^{3-p}}}
$$

The dilaton squared at the horizon is

$$
\varepsilon_{L}=\tilde{g}^{2}\left(1+\frac{u_{0}^{7-p}}{R^{7-p}}\right)\left(1+\frac{R^{7-p}}{u_{0}^{7-p}}\right)^{\frac{3-p}{2}}
$$


Thus the thermodynamics (41) and (42) is valid for NCOS theory when $\varepsilon_{D} \ll 1$ and $\varepsilon_{L} \ll 1$. This gives the phase structure of Section 1 with the only difference that $u$ is replaced with $u_{0}$. We have defined $\varepsilon_{D}$ and $\varepsilon_{L}$ for later convenience.

The thermodynamics (41) and (42) is clearly equivalent to that of OYM at strong 't Hooft coupling and with the identification $g_{\mathrm{YM}}^{2}=(2 \pi)^{p-2} \tilde{g}$. This is not surprising for energies $u_{0} \ll R$, since here the near-horizon solution (33)(36) is the usual near-horizon $\mathrm{D} p$-brane solution. For $u_{0} \gg R$ we get instead a prediction of the thermodynamics of NCOS theory.

If we consider the specific case of $p=3$ we know that for $u_{0}^{7-p} \sim N / \tilde{g}$ we go to the NCYM description which also has the thermodynamics (41) and (42) [12], so the thermodynamics is given by this whenever a supergravity description is available.

The fact that the thermodynamics does not change when raising the energy or temperature naturally raises the question about the stringy nature of NCOS theory. NCOS theory is claimed to be a string theory with a string spectrum and this should give rise to Hagedorn behaviour of the thermodynamics. Hagedorn behaviour occurs in a string theory for large temperatures and energies. When doing statistical mechanics of the string states the partition function can only be defined for temperatures lower than a certain maximal temperature, called the Hagedorn temperature. Near this temperature the thermodynamics behave in a singular manner. Clearly, the fact that (41) and (42) behaves uniformly for all energies means that there is no sign of any Hagedorn behaviour in this thermodynamics. Assuming of course $p \neq 5$ since for $p=5$ the thermodynamics (41) and (42) does exhibit Hagedorn behaviour, as we shall discuss further in Section 8 .

The Hagedorn temperature of NCOS theory is of order $T \sim 1$ since the string scale is equal to 1 . Thus, we should first test whether the temperature given in (41) can reach the Hagedorn temperature. That $T \sim 1$ is equivalent to $u_{0}^{5-p} \sim R^{7-p}$, or, equivalently,

$$
u_{0}^{5-p} \sim \tilde{g} N
$$

Thus, assuming $p<5$, we can see that if $\tilde{g} N \gg 1$ we need $u_{0} \gg 1$, and if $\tilde{g} N \ll 1$ we need $u_{0} \ll 1$. But, we know from Section 4 that if we want $\tilde{g} \ll 1 / N$ we must have $u_{0} \gg 1$, so that means that we cannot both be near the Hagedorn temperature and have arbitrarily weak coupling. This is a very

\footnotetext{
${ }^{10}$ See e.g. Appendix A in [26].
} 
important observation, since this can explain why the thermodynamics (41) and (42) does not exhibit Hagedorn behaviour. The explanation being that when $\tilde{g} \gg 1 / N$ strong coupling effects removes the Hagedorn behaviour of the string theory. This is perfectly possible in that the statistical derivation of Hagedorn behaviour assumes arbitrarily weak coupling. On the other hand, when $u_{0} \gg 1$ we can have arbitrarily weak coupling $\tilde{g} \ll 1 / N$ but this means that $T \gg 1$ so that we are far above the Hagedorn temperature. Thus, the Hagedorn temperature is clearly not limiting and we have a Hagedorn phase transition at $T \sim 1$.

The fact that we have the thermodynamics (41) and (42) for $\tilde{g} \gg 1 / N$ and $u_{0} \gg R$ suggests that the NCOS theory reduces to OYM in this region because of strong coupling effects.

In Section 7 we show that when approaching $u_{0} \sim 1$ for arbitrarily weak coupling new thermodynamics can occur. This supports our explanation of the missing Hagedorn behaviour in the thermodynamics.

\section{NCOS theory and delocalized F-strings}

In this section we elaborate on the fact that for $u \gg R$ the NCOS near-horizon solution from Section 3 reduce to that of the near-horizon limit of delocalized F-strings.

The solution for $M$ coincident F-strings delocalized in $p-1$ directions is

$$
\begin{gathered}
d s^{2}=\hat{H}^{-1}\left[-f d t^{2}+\left(d x^{1}\right)^{2}\right]+\left(d x^{2}\right)^{2}+\cdots+\left(d x^{p}\right)^{2}+f^{-1} d r^{2}+r^{2} d \Omega_{8-p}^{2} \\
e^{2 \phi}=\hat{H}^{-1} \\
B_{t 1}=\left(\hat{H}^{-1}-1\right) \operatorname{coth} \hat{\alpha}
\end{gathered}
$$

where we have used the functions and variables defined in Section 2. This is the supergravity solution that the F1-D $p$ bound state reduces to when $\hat{\theta}=\pi / 2$. From charge quantization of the F-strings we get

$$
r_{0}^{7-p} \cosh \hat{\alpha} \sinh \hat{\alpha}=\frac{(2 \pi)^{6} g_{s}^{2} l_{s}^{6}}{(7-p) V\left(S^{8-p}\right)} \frac{M}{V_{p-1}}
$$

This can also be gotten by combining (14) and (15). 
Taking the near-horizon limit $l_{s} \rightarrow 0$ with the variables in (29) and (30) fixed we get the near-horizon solution

$$
\begin{gathered}
\frac{d s^{2}}{l_{s}^{2}}=\frac{u^{7-p}}{R^{7-p}}\left[-f d \tilde{t}^{2}+\left(d \tilde{x}^{1}\right)^{2}\right]+\left(d \tilde{x}^{2}\right)^{2}+\cdots+\left(d \tilde{x}^{p}\right)^{2}+f^{-1} d u^{2}+u^{2} d \Omega_{8-p}^{2} \\
g_{s}^{2} e^{2 \phi}=\tilde{g}^{2} \frac{u^{7-p}}{R^{7-p}} \\
B_{t 1}=l_{s}^{2} \frac{u^{7-p}}{R^{7-p}}
\end{gathered}
$$

Thus we clearly see that the solution (33)-(36) reduces to the solution (52)-(54) for $u \gg R$.

The curvature of (52) and the dilaton (53) are small if and only we have

$$
1 \ll u^{7-p} \ll \frac{N}{\tilde{g}}
$$

If $\tilde{g} N \gg 1$ we need in addition that $u^{7-p} \gg \tilde{g} N$ in order to be describing NCOS theory. Thus, we need that $\tilde{g} \ll 1$.

From (15) we get that

$$
\tilde{g}=\frac{\tilde{V}_{p-1}}{(2 \pi)^{p-1}} \frac{N}{M}
$$

This is the connection between $M$ and $N$ which makes it possible to describe NCOS theory in terms of $M$ delocalized F-strings.

In [17, 18, 27] $\mathrm{D}(p-2)$-branes delocalized in two directions where shown to describe $p+1$ dimensional NCYM with a rank 2 non-commutative space at high energies. From this it was conjectured that the world-volume theory of the $\mathrm{D}(p-2)$-brane, which is OYM with $p-2$ space dimensions, is equivalent to the NCYM theory. It would be interesting to investigate if it is possible to make a similar connection for delocalized F-strings. The world-volume theory of $N$ coincident F-strings is the free orbifold CFT which is S-dual to OYM in $1+1$ dimensions 28 .

\section{Approaching new thermodynamics}

In this section we go beyond the leading order supergravity solution in order to find evidence that the thermodynamics of NCOS theory can differ from that of 
OYM. We choose to consider the special case $p=3$ only, but a similar analysis can be made for other dimensions.

The leading order type IIB supergravity receives string corrections of two types. Derivative corrections with expansion parameter $\alpha^{\prime}=l_{s}^{2}$ and loop corrections with expansion parameter $g_{s}$. These two types of expansions can be translated into two types expansions of the thermodynamics of the nearhorizon NCOS supergravity solution. Thus, the derivative expansion has the expansion parameter $\varepsilon_{D}$ given in (45), and the loop expansion has expansion parameter $\varepsilon_{L}$ given in (46) 凹. But, in order to use scaling arguments we need that the expansion parameters can be expressed as a constant times a power of $u_{0}$. Thus, we need either $u_{0} \ll R$ or $u_{0} \gg R$.

In type IIB string theory the first two corrections to the leading order supergravity action is the $l_{s}^{6} R^{4}$ term and the $g_{s}^{2} l_{s}^{6} R^{4}$ term. These will translate into a correction of order $\varepsilon_{D}^{3}$ and a correction of order $\varepsilon_{L} \varepsilon_{D}^{3}$.

If we consider the case $u_{0} \ll R$ which corresponds to 4 dimensional OYM we have

$$
\varepsilon_{D} \sim \frac{1}{\sqrt{\tilde{g} N}}, \quad \varepsilon_{L}=\tilde{g}^{2}
$$

Thus, we see that neither of the expansion parameters depends on $u_{0}$. This means for the OYM that the thermodynamics essentially stays the same when approaching $\varepsilon_{D} \sim 1$ or $\varepsilon_{L} \sim 1$. Thus, we will keep having the free energy $F \propto T^{4}$ but the coefficient in front will be renormalized $[2$.

If we instead consider the case $u_{0} \gg R$ which corresponds to 4 dimensional NCOS theory, we have

$$
\varepsilon_{D}=\frac{1}{u_{0}^{2}}, \quad \varepsilon_{L} \sim \frac{\tilde{g}}{N} u_{0}^{4}
$$

If we consider the transition point $\varepsilon_{L} \sim 1$, or, equivalently, $u_{0}^{4} \sim N / \tilde{g}$ we should not get any new thermodynamics since the theory should flow into 4 dimensional NCYM as described in 25, 4. Since NCYM also has $F \propto T^{4}$ the thermodynamics should be preserved when approaching this transition point. And, indeed, it can be shown [29] that the $\varepsilon_{L} \sim 1$ transition is very smooth due to the fact that the leading term that contributes around this point is of order $\varepsilon_{D}^{3} \varepsilon_{L}$ which is highly suppressed since $\varepsilon_{D} \ll 1$.

\footnotetext{
${ }^{11} \mathrm{~A}$ general account of how this works for various near-horizon backgrounds can be found in $[29$.

${ }^{12}$ For OYM there is a factor $3 / 4$ in difference between the free theory and the strongly coupled theory described by supergravity, as discussed in [30].
} 
On the other hand, if we consider the transition point $u_{0} \sim 1$, all the tree-level terms in type IIB string theory contribute. They form together a series of terms in the expansion parameter $\varepsilon_{D}=1 / u_{0}^{2}$, with the first term being $\varepsilon_{D}^{3}=1 / u_{0}^{6}$. Therefore we get new thermodynamics when approaching $u_{0} \sim 1$. This requires in fact $\tilde{g} \ll 1 / N$ since we still want $u_{0} \gg R$. This supports our arguments of Section 5 since we here stated that the Hagedorn behaviour should be observed in the region $u_{0}<1$ with very weak coupling $\tilde{g} \ll 1 / N$. We now see that exactly when we approach this region we get new thermodynamics.

From the arguments above, we note that the corrected free energy of 4 dimensional NCOS theory for $T$ approaching $1 /(\sqrt{\tilde{g} N})$ becomes

$$
F=-\frac{\pi^{2}}{8} N^{2} \tilde{V}_{3} T^{4}\left[1+\sum_{n=3}^{\infty} \frac{a_{n}}{(\tilde{g} N)^{n} T^{2 n}}\right]
$$

where $a_{n}$ are undetermined coefficients.

\section{The F1-D5 and D1-NS5 bound states}

We conclude this paper with a closer look at the $p=5$ case. This should correspond to NCOS theory in 6 dimensions, or, for low energies, 6 dimensional OYM. But, it is well-known that the D5-brane and NS5-brane world-volume theory is the so-called Little String Theory (LST) [31, 32, 33], which is a noncritical supersymmetric closed string theory in 6 dimensions. This means that NCOS theory in 6 dimensions is related to space-time non-commutative LST, and from this the question arises of how an open string theory can be related to a closed string theory on a non-commutative space-time. This question could be important for future research since we here have two non-critical string theories which are claimed to be descriptions of the same theory.

Our proposal for how NCOS theory and LST are related is that NCOS theory is a low energy limit of space-time non-commutative LST, since when we reach $u_{0}^{2} \sim N / \tilde{g}$ the theory flow to the near-horizon limit of the D1-NS5 bound state. Thus, we propose to define space-time non-commutative LST as the decoupling limit of D1-NS5, just as spatially non-commutative LST is defined as the decoupling limit of D2-NS5 or D3-NS5 [34]. And, we moreover propose that for sufficiently low energies this theory reduces to that of 6 dimensional NCOS theory. 
From (41) we get the Hagedorn temperature of the space-time non-commutative LST

$$
T_{\mathrm{LST}}=\frac{1}{2 \pi \sqrt{\tilde{g} N}}
$$

Thus, the LST Hagedorn temperature is different from that of NCOS theory, which confirms our suspicion that we have two different string scales in the theory.

Moreover, if we have $\tilde{g} \ll 1 / N$, which we expect is necessary in order to see the NCOS Hagedorn behaviour, we get that $T_{\mathrm{LST}} \gg 1$ so that any Hagedorn transition in NCOS theory would happen at much lower temperatures than $T_{\mathrm{LST}}$. If, on the other hand, $\tilde{g} N \gg 1$ we have that $T_{\mathrm{LST}} \ll 1$ which means that there cannot be any NCOS Hagedorn transition since the LST Hagedorn temperature is limiting 35, 36 and $T \sim 1$ thus cannot be reached.

The above consideration supports our proposal that NCOS theory is a low energy limit of space-time non-commutative LST since it means that whenever the NCOS Hagedorn temperature $T_{\mathrm{NCOS}}$ is defined we have that $T_{\mathrm{NCOS}} \ll T_{\mathrm{LST}}$.

Note also that the inverse string tension in LST is $\alpha_{\mathrm{LST}}^{\prime}=\tilde{g}$, while in NCOS theory it is $\alpha_{\mathrm{NCOS}}^{\prime}=1$. Thus, the low energy limit of LST is the limit $\tilde{g} \rightarrow 0$ which precisely is the limit where NCOS theory should be applicable since it is weakly coupled. Also, for $\tilde{g} \rightarrow 0$ we are in the F1-D5 description which corresponds to NCOS theory.

We now examine the LST Hagedorn behaviour of this theory using the arguments given in [35]. If we are at energies so that $u_{0} \gg R$ we have that

$$
\varepsilon_{D}^{3} \varepsilon_{L} \sim \frac{\tilde{g}}{N} \frac{1}{u_{0}^{2}}
$$

which gives

$$
S(T) \propto \frac{1}{T_{\mathrm{LST}}-T}
$$

This is valid for $u_{0}^{2} \ll N / \tilde{g}$. For $u_{0}^{2} \gg N / \tilde{g}$ we flow into the near-horizon limit of the D1-NS5 bound state. If we raise the energy sufficiently, we end up with a solution of D-strings delocalized in 4 directions. One can show that for these we get the thermodynamics

$$
S(T) \propto \frac{1}{\left(T_{\mathrm{LST}}-T\right)^{2 / 3}}
$$

valid for all sufficiently high energies. This is the same critical behaviour as for spatially non-commutative LST [35, 29]. It would be interesting if one could 
reproduce this from statistical arguments like it was done in [35, 36] for LST on a commutative space-time.

\section{Discussion}

In this paper we have studied NCOS theory using the dual supergravity description. We have presented the non-extremal F1-D $p$ bound state and its NCOS near-horizon limit. We have found that the thermodynamics to leading order is equivalent to that of OYM. We have furthermore argued that this does not have to be in contradiction with the expected Hagedorn behaviour of NCOS theory.

By considering string corrections to delocalized F-strings we found that the thermodynamics becomes very different from that of OYM when approaching the region with $u_{0}<1$ and very weak coupling $\tilde{g} \ll 1 / N$. This supports our conclusion that the supergravity thermodynamics is not in contradiction with the expectations from NCOS theory. The string correction analysis is also important since it is quantitative evidence that the thermodynamics only is equivalent to OYM at leading order in the supergravity. When string corrections are included the S-dual backgrounds of near-horizon D1-D3 and F1-D3 have different thermodynamics.

\section{Acknowledgments}

We thank N. Obers for useful discussions.

\section{References}

[1] A. Connes, M. R. Douglas, and A. Schwarz, "Noncommutative geometry and matrix theory: Compactification on tori," JHEP 02 (1998) 003, hep-th/9711162.

[2] N. Seiberg and E. Witten, "String theory and noncommutative geometry," JHEP 09 (1999) 032, hep-th/9908142.

[3] N. Seiberg, L. Susskind, and N. Toumbas, "Strings in background electric field, space / time noncommutativity and a new noncritical string theory," JHEP 06 (2000) 021, hep-th/0005040. 
[4] R. Gopakumar, J. Maldacena, S. Minwalla, and A. Strominger, "S-duality and noncommutative gauge theory," JHEP 06 (2000) 036, hep-th/0005048.

[5] N. Seiberg, L. Susskind, and N. Toumbas, "Space/time non-commutativity and causality," JHEP 06 (2000) 044, hep-th/0005015.

[6] J. L. F. Barbon and E. Rabinovici, "Stringy fuzziness as the custodian of time-space noncommutativity," Phys. Lett. B486 (2000) 202, hep-th/0005073.

[7] J. Gomis and T. Mehen, "Space-time noncommutative field theories and unitarity," hep-th/0005129.

[8] G.-H. Chen and Y.-S. Wu, "Comments on noncommutative open string theory: V-duality and holography," hep-th/0006013.

[9] J. Maldacena, "The large N limit of superconformal field theories and supergravity," Adv. Theor. Math. Phys. 2 (1998) 231-252, hep-th/9711200.

[10] N. Itzhaki, J. M. Maldacena, J. Sonnenschein, and S. Yankielowicz, "Supergravity and the large N limit of theories with sixteen supercharges," Phys. Rev. D58 (1998) 046004, hep-th/9802042.

[11] O. Aharony, S. S. Gubser, J. Maldacena, H. Ooguri, and Y. Oz, "Large N field theories, string theory and gravity," Phys. Rept. 323 (2000) 183, hep-th/9905111.

[12] J. M. Maldacena and J. G. Russo, "Large N limit of non-commutative gauge theories," JHEP 09 (1999) 025, hep-th/9908134.

[13] A. Hashimoto and N. Itzhaki, "Non-commutative Yang-Mills and the AdS/CFT correspondence," Phys. Lett. B465 (1999) 142, hep-th/9907166.

[14] M. Li and Y.-S. Wu, "Holography and noncommutative Yang-Mills," Phys. Rev. Lett. 84 (2000) 2084, hep-th/9909085. 
[15] M. Alishahiha, Y. Oz, and M. M. Sheikh-Jabbari, "Supergravity and large N noncommutative field theories," JHEP 11 (1999) 007, hep-th/9909215.

[16] T. Harmark and N. A. Obers, "Phase structure of non-commutative field theories and spinning brane bound states," JHEP 03 (2000) 024, hep-th/9911169.

[17] J. X. Lu and S. Roy, " $(p+1)$-dimensional noncommutative Yang-Mills and D $(p-2)$ branes," Nucl. Phys. B579 (2000) 229, hep-th/9912165.

[18] R.-G. Cai and N. Ohta, "Noncommutative and ordinary super Yang-Mills on (D $(p-2), \mathrm{D} p)$ bound states," JHEP 03 (2000) 009, hep-th/0001213.

[19] M. S. Costa, "Black composite M-branes," Nucl. Phys. B495 (1997) 195, hep-th/9610138.

[20] M. B. Green, N. D. Lambert, G. Papadopoulos, and P. K. Townsend, "Dyonic p-branes from self-dual (p+1)-branes," Phys. Lett. B384 (1996) 86-92, hep-th/9605146.

[21] J. G. Russo and A. A. Tseytlin, "Waves, boosted branes and BPS states in M-theory," Nucl. Phys. B490 (1997) 121-144, hep-th/9611047.

[22] M. S. Costa and G. Papadopoulos, "Superstring dualities and p-brane bound states," Nucl. Phys. B510 (1998) 217, hep-th/9612204.

[23] J. X. Lu and S. Roy, "Non-threshold (F,Dp) bound states," Nucl. Phys. B560 (1999) 181, hep-th/9904129.

[24] C. M. Hull, "Timelike T-duality, de Sitter space, large N gauge theories and topological field theory," JHEP 07 (1998) 021, hep-th/9806146.

[25] O. J. Ganor, G. Rajesh, and S. Sethi, "Duality and non-commutative gauge theory," hep-th/0005046.

[26] T. Harmark and N. A. Obers, "Thermodynamics of spinning branes and their dual field theories," JHEP 01 (2000) 008, hep-th/9910036.

[27] D. Youm, "A note on (D(p-2),Dp) bound state and noncommutative Yang-Mills theory," hep-th/0006019. 
[28] R. Dijkgraaf, E. Verlinde, and H. Verlinde, "Matrix string theory," Nucl. Phys. B500 (1997) 43-61, hep-th/9703030.

[29] J. Correia, T. Harmark, and N. A. Obers. In preparation.

[30] S. S. Gubser, I. R. Klebanov, and A. A. Tseytlin, "Coupling constant dependence in the thermodynamics of $\mathrm{N}=4$ supersymmetric Yang-Mills theory," Nucl. Phys. B534 (1998) 202, hep-th/9805156.

[31] N. Seiberg, "New theories in six-dimensions and matrix description of M theory on $T^{5}$ and $T^{5} / Z_{2}$," Phys. Lett. B408 (1997) 98-104, hep-th/9705221.

[32] M. Berkooz, M. Rozali, and N. Seiberg, "Matrix description of M theory on $T^{4}$ and $T^{5}, "$ Phys. Lett. B408 (1997) 105-110, hep-th/9704089.

[33] A. Losev, G. Moore, and S. L. Shatashvili, "M \& m's," Nucl. Phys. B522 (1998) 105-124, hep-th/9707250.

[34] M. Alishahiha, "On type II NS5-branes in the presence of an RR field," hep-th/0002198.

[35] T. Harmark and N. A. Obers, "Hagedorn behaviour of little string theory from string corrections to NS5-branes," Phys. Lett. B485 (2000) 285 , hep-th/0005021.

[36] M. Berkooz and M. Rozali, "Near Hagedorn dynamics of NS fivebranes, or a new universality class of coiled strings," JHEP 05 (2000) 040, hep-th/0005047. 